\title{
Energy Efficiency Gains Using VHOs in Heterogeneous Networks
}

\author{
António Serrador and Luís M. Correia \\ Instituto Superior Técnico/Instituto de Telecomunicações, \\ Technical University of Lisbon, Portugal R. Rovisco Pais, 1049-001 Lisbon \\ \{ajns, luis.correia\}@lx.it.pt
}

\begin{abstract}
Partially, the future of mobile and cellular networks will be about energy consumption savings, at the network infrastructure side. In the heterogeneous cellular networks environment, these can be achieved based on bandwidth adaptation, discontinuous transmission, relays, or even Base Stations (BSs) shutdown when possible. This paper proposes an approach for power savings using Vertical Handovers (VHOs) among different Radio Access Technologies (RATs), based on Radio Resource Management. The presented solution use a utility function focused on the energy efficiency of a given BS and RAT. An active VHO trigger mechanism is also proposed to provide RF power savings by handover mobile terminals to the most efficient RAT. Based on a power model proposed by the ICT-EARTH project, it is possible to translate RF power savings into system level ones. Results show that VHOs RF power gains range up to $46 \%$, and up to $13 \%$ at the BS system level.
\end{abstract}

Keywords: Energy Efficiency, Radio Resource Management, Vertical Handover, Heterogeneous Networks.

\section{Introduction}

In wireless mobile heterogeneous network environments, Radio Resource Management (RRM) and Joint RRM (JRRM) entities, and their corresponding functionalities/algorithms, can be used to manage modern networks focused on different goals. One of them is definitely networks infrastructures energy consumption, and the corresponding impact on networks operators cost, such the Operational Expenditure (OPEX), simultaneously addressing the carbon dioxide emissions problem. Nowadays, the whole Information and Communication Technologies (ICT) industry [1]-[2] is responsible for about $2 \%$ of $\mathrm{CO}_{2}$ emissions worldwide, energy cost ranging from 20 to $35 \%$ of operators OPEX. Thus, it is very important to decrease power consumptions in all areas, using imaginative techniques.

In recent literature and research projects, Vertical Handovers (VHOs) are used to save energy in Mobile Terminals (MTs), especially the new 4G ones. In [3], authors use VHOs between IEEE 802.11 and 802.16 e systems to reduce terminals power consumption. Detailed power and performance models for both standards are defined to evaluate the VHO opportunity. More recently, European projects are dedicated to 
Energy Efficiency (EE) in mobile networks: the EARTH project [1] is devoted to reduce networks infrastructure power consumption, using a wide set of saving techniques; C2POWER [4], addresses EE optimisation in MTs, assuming multi-Radio Access Technologies (RATs) capabilities, attaching MTs to the most efficient radio link. Authors in [5] also address VHOs as a fundamental technique, by proposing a RAT selection algorithm that selects a RAT with lower energy consumption to increase terminal battery life, while respecting QoS. In [6], authors propose EE VHOs by reducing MTs frame overhead. Nevertheless, relevant network infrastructure EE using VHOs is not addressed.

In this work, VHOs are used to provide additional EE gains in the network infrastructure and not only at MTs. The idea is as follows: in a multi-RAT environment, legacy RATs are usually less energy efficient when comparing power and information processing, thus, moving active MTs (and corresponding services) to modern RATs provides additional EE, assuring a reasonable QoS balance among RATs. Aiming at this goal, implies taking three steps: the EE metric is identified and used in a Cost Function (CF); a VHO trigger algorithm is implemented and proposed; a system level power model is computed to obtain the overall energy gain in a given Base Station (BS), knowing the radio power gain.

This approach assumes the existence of a JRRM entity in a very tight coupling architecture, being simulated by a multi-RAT simulator tool [7], capable of simulating radio interfaces such as Wideband Code Division Access (WCDMA) and High Speed Downlink Packet Access (HSDPA) defined by the $3^{\text {rd }}$ Generation Partnership Project (3GPP); also, traffic source models are implemented, such as voice, web browsing and File Transfer Protocol (FTP). By using this tool, micro-cells can be simulated in an urban dense scenario.

The main goal and novelty of this paper is to obtain the EE gains at radio and BS system levels, by exploring EE margins in a multi-RAT environment, using VHOs and relevant metrics to trigger handovers.

The paper is structured as follows. Section 2 presents the BSs power blocks and the power relation between RF and system levels. In Section 3, the used metrics, CFs and VHOs algorithm are proposed, while in Section 4, the fundamental reference scenario is presented for micro-cells and corresponding traffic loads, computational results being discussed. Finally, conclusions are drawn in Section 5.

\section{BS Power Model}

This section defines the BS micro-cell power model consumption defined by the EARTH project 8. It is assumed that the 2010 BSs systems state of the art power conditions are available. This model is relevant because it enables the computation of system level power gains, knowing the RF EE gain provided by the use of VHOs or another RRM technique. Thus, the model described in this section is used to estimate the BS complete system power gains, knowing the RF power savings provided by techniques or algorithms running at the air interface.

The power consumption of BSs is dominated by the radio supporting equipment. In general macro-, micro-, pico- and femto-cells BS equipment blocks include 
multiple transceivers (TRXs) with multiple antennas. Each of these TRXs comprises a loss Antenna Interface (AI), a Power Amplifier (PA), a Radio Frequency (RF) small-signal transceiver section and a baseband interface (BB) including both a receiver (UL) and a transmitter (DL) section, a DC-DC power supply regulation, an active cooling system, and finally a main AC-DC power supply for connection to the electrical power grid. Note that active cooling is only relevant for a macro-cell BS, being negligible for smaller cells. Details about this model are provided in 8 .

In Fig. 1, the BS overall power consumption dependency on RF output power is highlighted.

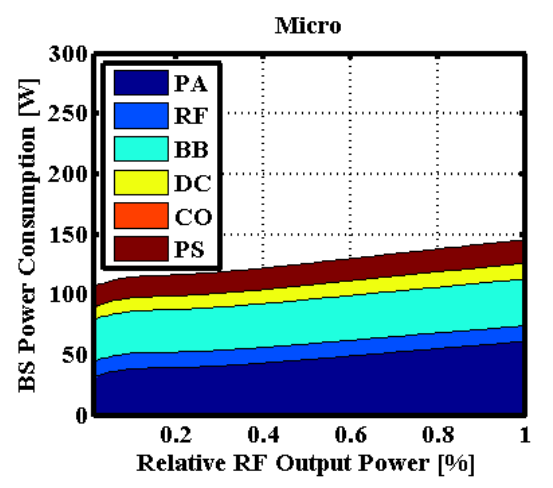

Fig. 1. Micro-cell RF and BS power relation (extracted from [8])

The EARTH project proposes a simple relation between RF and BS power as follows:

$$
P_{B S}=P_{0}+\Delta_{P} \cdot P_{R F}, \quad 0 \leq P_{R F} \leq P_{\max }
$$

where,

- $\quad P_{B S}$, is the overall required BS power;

- $\quad P_{R F}$, is the output RF power;

- $\quad P_{o}$, is the BS power consumption calculated at the minimum possible RF power;

- $\quad \Delta_{P}$, is the linear $P_{B S}$ relation with $P_{R F}$, for each BS type;

- $\quad P_{\max }$, is the maximum RF power.

\section{JRRM and RRM Models}

\subsection{EE Metrics and Cost Function}

In general, transmission of user data is accompanied by additional network signalling. In some cases, even in an "empty" network, radio beacons transporting network information must be continuously transmitting useful data (e.g., BS identification) to standby MTs. Moreover, user data includes other information, such as, radio channel formatting and coding overhead, high network layers protocol additional information, and retransmission of information when radio channels are of poor quality. Therefore, in the end, the relation 
between real user data and overhead is relevant when discussing EE, and the relation between transmitted bit and power. One can divide the previous concepts into the following parameters, Fig. 2:

- The information volume related to overhead and signalling (includes network and users signalling data) issues, as well as retransmissions, $V_{O}$;

- The information volume related only to user real data generated by the application, $V_{D}$;

- The total information volume $V_{T}$, required to transmit/support the user data, is $V_{O}$ plus $V_{D}$.

- The power required to transmit the overhead information $V_{O}$, including network and users signalling data, is $P_{O}$;

- The power required to transmit user information $V_{D}$ is defined as $P_{D}$;

- The total power required to transmit $V_{T}$ is assumed to be $P_{T}$.

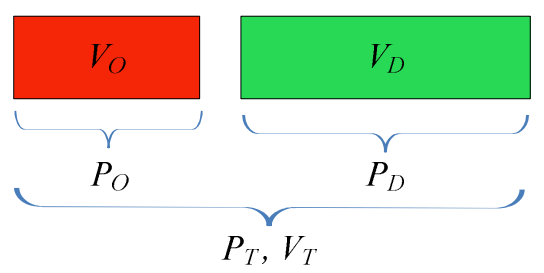

Fig. 2. Network and users' power and information parameters

Additionally, the BS energy cost can be established, being assumed as an EE metric of a given BS and associated RAT. Thus, the total power cost required to transmit $V_{D}$, is assumed to be $C_{U}$, given by:

$$
C_{U[\mathrm{w} / \mathrm{bit}]}=\frac{P_{T[\mathrm{w}]}}{V_{D[\mathrm{bit}]}}
$$

This power $\operatorname{cost} C_{U}$ is a key metric to measure the EE of a given BS; however, appropriate RRM decisions should be based on an opportunistic time scale, being crucial to evaluate BSs efficiency, therefore, $C_{U}$ can also be measure in W/bps. This metric is computed by a CF defined in 9 and 10, being used in this work only with the operator component. Different CFs may be computed for each different RAT type. Each one of these "sub CFs" are weighted with different values, enabling the implementation and evaluation of different policies by RRM algorithms over each type of RAT. The CF model can be used at different network levels as follows: network KPIs (Key Performance Indicators), MTs, BSs, RATs, and operators. In order to combine all these different parameters, they must be normalised. For operators, the total cost for a given RAT $r, C o_{r}$, is calculated as follows:

$$
C o_{r}=\frac{1}{N_{B S_{r}}} \sum_{b=1}^{N_{B S_{r}}} C o_{r, b}
$$

where, 
- $N_{B S r}$ is the total number of BSs for a given RAT $r$,

- $C o_{r, b}$ is the operator's cost for each BS $b$, in RAT $r$, being computed by:

$$
C o_{r, b}=\frac{1}{\sum_{i=1}^{N_{K P I} r} w_{r, i}} \sum_{i=1}^{N_{K P I r} r} w_{r, i} \cdot k_{b, i}
$$

where,

- $N_{K P I r}$ is the total number of KPIs of a given RAT $r$;

- $w_{r, i}$ is the weight of each KPI $i$;

- $k_{b, i}$ represents the normalised value of each KPI $\left(0 \leq k_{b, i} \leq 1\right)$.

Note that KPIs can be any relevant parameter that is useful for the management of cellular networks. In this paper, the power $\operatorname{cost} C_{U}$ metric is used as a KPI. Modern RRM algorithms can use this CF model or principles to guide networks focused on their EE metrics (among others), providing fast methods to distinguish the most efficient BSs and RATs at different networks operation time scales.

\subsection{RRM Algorithms}

In 11, an algorithm to trigger VHOs is proposed, the so called Fittingness Factor (FF). This algorithm is divided into two parts, for new connections, and for on-going ones. An integrated parameter is defined by the FF algorithm, the final cost factor $F_{u, p, s, r}$, which reflects the degree of adequacy of a given BS/RAT to a given user: it takes each cell of the $r$ RAT for each $u$ user, belonging to the $p$ customer profile, requesting a given $s$ service. The RAT selection algorithm is considered differently, depending on whether the selection is done at session set-up or during an on-going connection.

For a user requesting service $s$, Fig. 3, the procedure is:

- Measure the $F_{u, p, s, r}$ for each candidate cell $k_{r}$ of the $r$ detected RAT.

- Select the RAT $r$ having the cell with the highest $F_{u, p, s, r}$ among all candidate cells:

$$
R=\underset{r}{\arg \max }\left(\max _{k_{r}} F_{u, p, s, r}\left(k_{r}\right)\right)
$$

In case that two or more RATs have the same $F_{u, p, s, r}$ value, then, the less loaded RAT is selected.

- Run the Call Admission Control (CAC) procedure in the RAT $r$. If admission is not possible, try the next RAT in decreasing order of $F_{u, p, s, r}$, provided that its $F_{u, p, s, r}$ value is higher than 0 . If no other RATs with $F_{u, p, s, r}$ higher than 0 exist, block the call.

For on-going connections, Fig. 4, the proposed criterion to execute a VHO algorithm is based on the FF. It assumes that the MT is connected to the RAT denoted as "servingRAT" and cell denoted as "servingCell". 


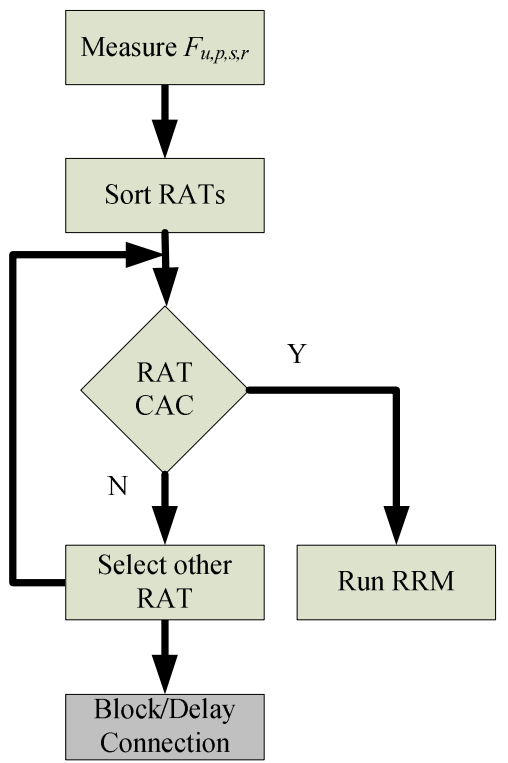

Fig. 3. FF BS/RAT selection algorithm (new connections)

In this case of on-going connections, the procedure is:

- For each candidate cell and RAT, monitor the corresponding $F_{u, p, s, r}\left(k_{r}\right)$. Measures should be averaged during a period $T$.

- If condition

$$
F_{u, p, s, r}\left(k_{r}\right)>F_{u, p, s, \text { servingR } A N}(\text { servingCell })+\Delta_{\text {VHO }}
$$

holds during a period $T_{V H O}$, then a VHO to RAT $r$ and cell $k_{r}$ should be triggered, provided that there are available resources for the user in this RAT and cell.

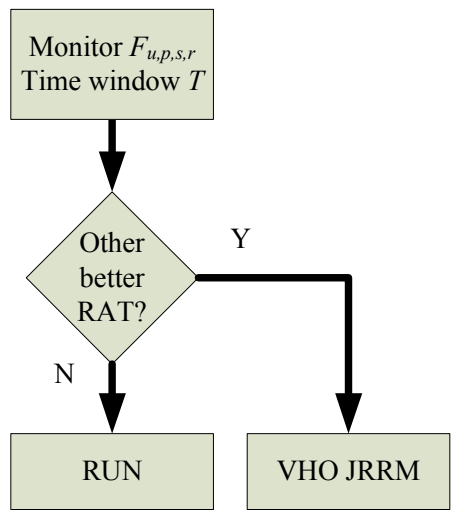

Fig. 4. The FF VHO triggering process (on-going connections) 


\section{Scenario and Results}

\subsection{Reference Scenario}

In order to assess the impact on EE performance produced by the previous algorithms, micro-cell BS power and traffic models are used to design a reference scenario proposed in 8 .

As mentioned previously, the BS equipment power model consumption corresponds to a typical commercially BS in 2010, used in this work as reference for simulations and for system level EE gains. Table 1 presents values for the BS, implementing an approximation to Fig. 1; the BS system power levels are computed via (1).

Table 1. Model Parameters for Micro-Cells

\begin{tabular}{c|c|c|c}
\hline \hline BS type & $P_{\max }[\mathrm{W}]$ & $P_{0}[\mathrm{~W}]$ & $\Delta_{P}$ \\
\hline \hline Micro & 6.3 & 106 & 6.35 \\
\hline \hline
\end{tabular}

Different services are also under test, because different RATs present different radio bearers, since they handle services in a different manner (e.g., voice in circuit switch, or data in packet switch networks). The reference number assumed for the users' density is $1000 \mathrm{user} / \mathrm{km}^{2}$, which corresponds to a Low (L) traffic case; in order to assess the traffic load impact on EE, users density is increased by 25 and $50 \%$, corresponding to Medium $(\mathrm{M})$ and High $(\mathrm{H})$ traffic load cases, respectively. In the reference scenario, one assumes two BSs, WCDMA and HSDPA, with a $500 \mathrm{~m}$ radius, WCDMA being considered the legacy RAT in which EE may be enhanced. The considered service set is defined according to Table 2, where reference parameters are provided for each service, arrival rate $\lambda$, session time $\tau$ or volume $V_{D}$ being defined. The average number of active users defined in the simulator setting is valid for the Low traffic situation and for both BSs.

Table 2. Services and Traffic (Reference)

\begin{tabular}{l|c|c|c|r}
\hline \hline Services & $\begin{array}{c}\text { Average number } \\
\text { of active users }\end{array}$ & $\lambda$ & $\begin{array}{c}\tau \\
{[\mathrm{s}]}\end{array}$ & $\begin{array}{c}V_{D} \\
{[\mathrm{MB}]}\end{array}$ \\
\hline \hline Voice & 15 & 0.31 & 90 & - \\
\hline WWW & 6 & 0.5 & - & 0.1 \\
\hline FTP & 6 & 0.1 & - & 10 \\
\hline \hline
\end{tabular}

\subsection{Results}

The first step to obtain EE gains starts by computing the BS RF power level, and comparing two distinguished cases: the previous EE algorithms being OFF and ON. 
Based on the reference scenario, different simulations were performed, RF power results being presented in Table 3, where each service is individually evaluated (active users are performing only this particular service), as well for the case where all services are combined.

Table 3. RF Power Levels and Services Traffic Load

\begin{tabular}{c|c|c|c|c}
\multicolumn{4}{c}{} & \multicolumn{4}{c}{$\begin{array}{r}\text { WCDMA RF Power [W] } \\
\text { Traffic Load Profiles }\end{array}$} \\
\hline \hline Services & EE [ON/OFF] & Low & Medium & High \\
\hline \hline \multirow{2}{*}{ Voice } & OFF & 1.9 & 2.9 & 2.9 \\
\cline { 2 - 5 } & ON & 1.4 & 1.5 & 1.9 \\
\hline \multirow{2}{*}{ WWW } & OFF & 4.5 & 4.9 & 5.3 \\
\cline { 2 - 5 } & ON & 2.4 & 3.0 & 5.2 \\
\hline \multirow{2}{*}{ FTP } & OFF & 3.3 & 3.5 & 5.4 \\
\cline { 2 - 5 } & ON & 2.3 & 2.7 & 5.0 \\
\hline \multirow{3}{*}{ ALL } & OFF & 6.8 & 6.6 & 6.2 \\
\cline { 2 - 5 } & ON & 3.8 & 5.3 & 6.0 \\
\hline \hline
\end{tabular}

The EE gain results for WCDMA $G_{R F}$, at the radio level, are presented by Fig. 5. Corresponding results, using the previous power model, at BS system level, $G_{S L}$, are presented in Fig. 6. $G_{R F}$ is calculated by comparing results were the FF is OFF and ON; by using (1), $G_{S L}$ is computed in the same way. By observing Fig. 5, one concludes that only the voice service increases its EE for the Medium load case. This happens because voice has less impact on the network load (compared to data), providing a margin to increase EE even in Medium load; however for High load, voice users trigger VHOs, providing a lower EE gain.

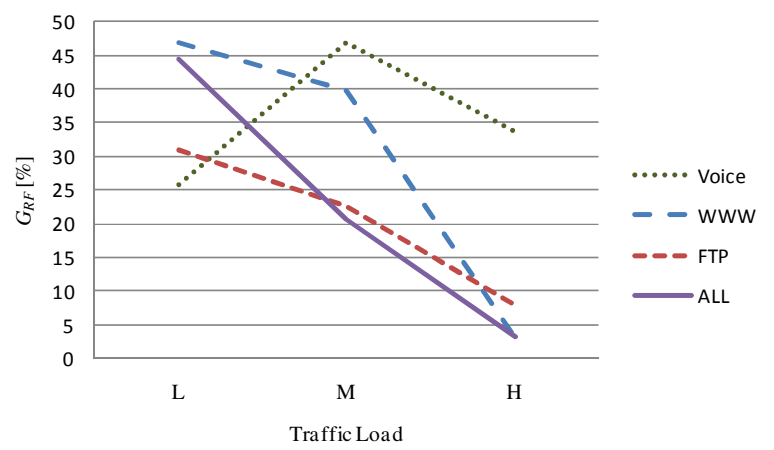

Fig. 5. RF EE gains for each simulated case 
When traffic rises, EE gain decreases, because the number of VHOs triggered by the FF also decreases, due to the lower power margin offered by multi-RAT BSs. The only exception is voice, since it takes less radio BSs resources, thus, networks can offer EE gains even when voice traffic increases (e.g., Medium load). Other effects become important, e.g., handover receiving RATs become more loaded, therefore, less power efficient, since more intra-cell interference becomes also significant. Another effect is distance, since HSDPA networks, by comparison with legacy ones, may become less power efficient for long range communications, or may reduce the coverage of high bit rate services, leading potential VHO situations to be less interesting or impossible. For the WWW and FTP services, differences on EE gains curves are due to their session density, duration, or burstiness intrinsic natures. The results from Fig. 6 follow the previous RF gains, however, for the same cases the effect of the $\Delta P$ factor can be observed; for example, in the Low case, when all services are combined, it presents higher gains compared to the single WWW case. Anyway, for the all services case, EE ranges from about 1 up to $13 \%$, which means that one can cut $13 \%$ in $\mathrm{WCDMA} \mathrm{CO}_{2} \mathrm{BSs}$ emissions and network operation costs.

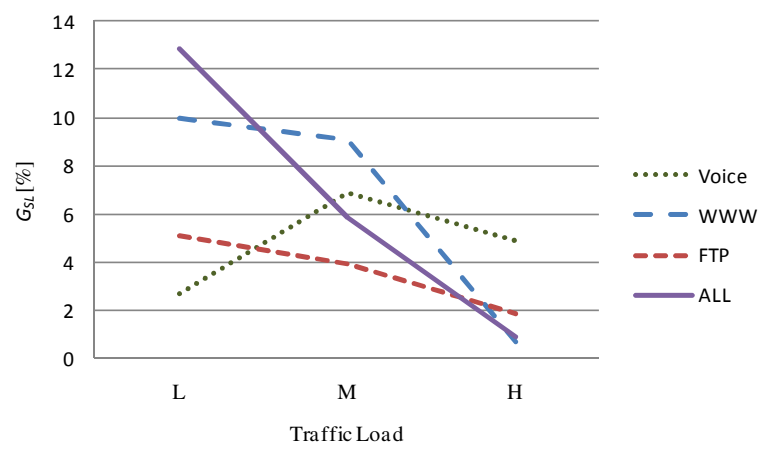

Fig. 6. EE at system level gains for each simulated case

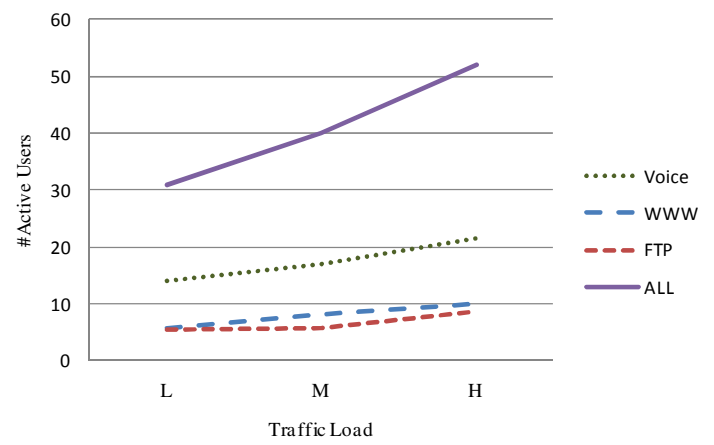

Fig. 7. Average number of active MTs/service generated 
When all services are simulated simultaneously, the RF EE gain decreases with the traffic load, but it can achieve relatively high gains in the Low traffic case, because, in this traffic scenario, VHOs have some power margin, producing EE gains (being coherent with WWW and FTP trends).

Fig. 7 shows the total average number of active users generated by the tool for both BSs under simulation and for all previous situations. The FTP and WWW results are very similar as expected. These results confirm the previous effects, since it is shown that real-time services rise according to the plan defined in the scenario.

\section{Conclusions}

This paper proposes VHOs to be used as one more technique in the battle for power consumption reduction in mobile cellular infrastructures, being applicable in a multiRAT environment. Simulations include propagation issues, services and traffic models and JRRM algorithms to trigger VHOs guided by EE metrics, such as W/bps. The capability to distinguish packets overhead and users' data is also included.

The model gives EE gains directly at the RF level, using the presented BS power model, being possible to compute EE gains at the BS system level.

Results for voice, web browsing, and file transfer show EE gains at RF levels ranging from 5 up to $46 \%$, for High and Low traffic cases, respectively.

EE gains for micro-cell BS power consumption, at the system level, range from 1 to $13 \%$, achieved for High and Low traffic cases, respectively. Thus, one may conclude that in very high traffic load conditions, VHOs will have a very short margin to decrease power consumptions, since BSs will be closer to congestion. Nevertheless, for Low and Medium traffic loads, EE gains present interesting values that should be considered.

Acknowledgements. The research leading to these results has received funding from the European Community's Seventh Framework Programme FP7/2007-2013 under grant agreement $n^{\circ} 247733$ - project EARTH.

\section{References}

1. http: //www.ict-EARTH.eu/ (February 2011)

2. Ericsson, Sustainable energy use in mobile communications, White paper (August 2007), http://www.ericsson.com/campaign/sustainable_mobile_communic ations/downloads/sustainable_energy.pdf

3. Desset, C., Ahmed, N., Dejonghe, A.: Energy savings for wireless terminals through smart vertical handover. In: Proc. of ICC 2009 - 2009 IEEE International Conference on Communications, Dresden, Germany (June 2009), http : / /www. ieee-icc . org / 2009

4. Radwan, A., Rodriguez, J., Gomes, A., Sá, E.: C2POWER Approach for Power Saving in Multi-standard Wireless Devices. In: Proc. of VTC 2011 Spring - 73rd IEEE Vehicular Technology Conference, Budapest, Hungary (May 2011),

http: //www. ict-c2power. eu 
5. Kanno, I., Yamazaki, K., Ikeda, Y., Ishikawa, H.: Adaptive Energy Centric Radio Access Selection for Vertical Handover in Heterogeneous Networks. In: Proc. of WCNC $2010-$ IEEE Wireless Communications and Networking Conference, Sydney, Australia (April 2010), http: / /www. ieee-wcnc. org/2010

6. Seo, S., Song, J.S.: Energy-Efficient Vertical Handover Mechanism. IEICE Transactions on Communications E92-B(9), 2964-2966 (2009)

7. Serrador, A. (ed.): Simulation tools: final version capabilities and features, IST-AROMA Project, Del. D13, EC-IST Office, Brussels, Belgium (April 2007),

http: / / www . aroma-ist. upc. edu

8. Imran, M.A., Katranaras, E. (eds.): Energy efficiency analysis of the reference systems, areas of improvements and target breakdown. ICT-EARTH Project, Deliverable D2.3, EC-IST Office, Brussels, Belgium (January 2011), http: / / www . ict-EARTH . eu

9. Serrador, A., Correia, L.M.: A Cost Function Model for CRRM over Heterogeneous. Wireless Personal Communications 59(2), 313-329 (2011), http: / /www. springer. com/engineering/signals/journal/11277

10. Serrador, A., Correia, L.M.: Policies For a Cost Function For Heterogeneous Networks Performance Evaluation. In: Proc. of PIMRC 2007 - 18th Annual IEEE International Symposium on Personal Indoor and Mobile Radio Communications, Athens, Greece (September 2007), http: / /www . pimrc2007. org

11. Pérez-Romero, J.: Operator's RAT Selection Policies Based on the Fittingness Factor Concept. In: Proc. of 16th IST Mobile and Wireless Communications Summit, Budapest, Hungary (June 2007) 